\title{
DER DEUTSCHE SOCIAL-DEMOKRATISCHE ARBEITERVEREIN IN KOPENHAGEN UND DIE DÄNISCHE ARBEITERBEWEGUNG
}

Von den verschiedenen Auslandsorganisationen sozialdemokratischer deutscher Arbeiter und Handwerker im neunzehnten Jahrhundert sind die drei Kopenhagener, der Deutsche social-demokratische Arbeiterverein (1875-77), die Deutsche Lesegesellschaft (1887-91) und der Deutsche Arbeiterverein Vorwärts (1894-1914) bislang von der historischen Forschung unbeachtet geblieben. Dies erscheint unberechtigt, denn, mag es auch zutreffen, dass die drei Vereine zu den weniger bedeutenden Auslandsorganisationen deutscher Sozialdemokraten gehörten, ${ }^{1}$ für die dänische Arbeiterbewegung war der älteste der drei Vereine von grosser Bedeutung. In der Mitte der siebziger Jahre des vorigen Jahrhunderts war er nicht nur das einzige Bindeglied der dänischen Arbeiterbewegung zwar weniger zur organisierten deutschen Sozialdemokratie als zur Ideenwelt deutscher Arbeiter und Handwerker, sondern zeitweilig auch das einzige Milieu innerhalb der dänischen Arbeiterbewegung, in dem theoretische Fragen diskutiert wurden. Die Aufgabe, diesen Verein darzustellen, erschien deshalb lohnend; dabei erwies es sich als notwendig, ihn in die Geschichte der dänischen Arbeiterbewegung, in die er voll integriert war, einzubetten.

Den durch Qualität und Quantität des vorhandenen Quellenmaterials bedingten Eindruck des Skizzenhaften kann die Darstellung nicht verleugnen. So ist der wichtigste Teil der Selbstzeugnisse des Vereins, Sitzungsprotokolle und „Mitgliedsbüchlein”, unauffindbar und wahr-

${ }^{1}$ Dies trifft in jedem Falle für den Deutschen Arbeiterverein Vorwärts zu; das umfangreiche Quellenmaterial im ABA (Arbejderbevægelsens Bibliotek og Arkiv) in Kopenhagen (6 Bände Protokolle der Vorstandssitzungen, 1 Bücherverzeichnis der Vereinsbibliothek sowie deren Kassabuch) suggeriert das Bild eines kleinbürgerlichen Geselligkeitsvereins. Zur Deutschen Lesegesellschaft sind die Quellen allerdings sehr spärlich. Nachforschungen in den dänischen Archiven sowie in der sozialdemokratischen Presse Dänemarks blieben ohne Ergebnis, doch werden im Nachlass Julius Motteler im Internationaal Instituut voor Sociale Geschiedenis eine Reihe von Briefen aufbewahrt, die die Existenz der Lesegesellschaft im genannten Zeitraum bezeugen ( $\mathrm{Nr} 358,592,866,1250$ und 1258). 
scheinlich verlorengegangen. Ausserdem hat die ansonsten sehr aufmerksame dänische Polizei den deutschen Verein nicht registriert, ${ }^{1}$ ebensowenig wie die deutsche Botschaft in Kopenhagen, die in anderen Fällen regelmässige Berichte über das Treiben der dänischen Sozialisten nach Berlin schickte. ${ }^{2}$ Unsere Darstellung fusst deshalb hauptsächlich auf den - allerdings sehr zahlreichen - Berichten und Notizen in der dänischen und deutschen sozialdemokratischen Presse sowie auf dem einzigen erhaltengebliebenen Exemplar der Satzungen des Vereins (Dok. I).

Am Donnerstag dem 9. September 1875 war in Social-Demokraten, dem Organ der dänischen Gewerkvereine, unter der Überschrift „,Tysk Foredrag" (Deutscher Vortrag) in dänischer Sprache die folgende Notiz zu lesen:

Am Sonnabend dem 11. September abends $8 \frac{1}{2}$ Uhr, hält der Unterzeichnete einen deutschen Vortrag in Lille Kongensgade $\mathrm{Nr} 25$ [Kopenhagen] über das Thema: Die sozialistischen Grundsätze und die Arbeiterbewegung draussen und hierzulande. Hierzu werden alle deutschen Arbeiter eingeladen! Eintritt nach Belieben, welcher dem Agitationsfond [der dänischen Gewerkvereine] zufällt.

E. W. Klein. ${ }^{3}$

Über den angekündigten Vortrag, der tags darauf um 24 Stunden vorgezogen und an einen anderen Ort verlegt wurde, ${ }^{4}$ berichtete Social-Demokraten in einem ausführlichen Referat. Die Veranstaltung sei von ,zahlreichen deutschen als auch dänischen Gesinnungsgenossen” besucht worden; der Initiator E. W. Klein habe im Laufe seiner Ausführungen bemängelt, dass in der dänischen Hauptstadt viele deutsche Sozialisten wohnten und arbeiteten, die aber wegen mangelnder Kenntnisse der dänischen Sprache „völlig davon ausgeschlossen seien, an der sozialistischen Bewegung teilzunehmen". Er habe deshalb den Entschluss gefasst, einen „deutschen sozialdemokratischen

1 Recherchen in Politidirektørens Arkiv, Landsarkivet for Sjælland, Kopenhagen, blieben ergebnislos.

2 Politisches Archiv des Auswärtigen Amtes, Bonn, Europa Generalia, Nr 39 : Akten betreffend die Herstellung einer engen Verbindung unter den konservativen Monarchien gegen republikanisch-sozialistische Bestrebungen, Bde 6-9 (1873-77).

3 Social-Demokraten (weiter zitiert als: Soc.-Dem.), 2. Jahrg., Nr 208 (9.9.1875).

- Ebd., Nr 209 (10.9.1875). 
Verein" zu gründen. ${ }^{1}$ Laut Referat hatte Klein sich vorgestellt, dass der Verein für die deutschen Arbeiter und Handwerker in Kopenhagen Sprachkurse veranstalten und ein Arbeitsvermittlungsbüro für Ausländer in der dänischen Hauptstadt unterhalten könnte. ${ }^{2}$ Weitere Einzelheiten über die Ziele des annoncierten Vereins teilt das Referat nicht mit. Anschliessend an Kleins Referat hielt der Deutsche Simonsen einen Vortrag über die Sozialdemokratie im deutschen Reichstag, und danach wählten die versammelten deutschen Arbeiter und Handwerker aus ihrer Mitte eine Kommission, die die Statuten des geplanten Vereins erarbeiten sollte. Die Kommission bestand aus den beiden Referenten und ihren Landsleuten Rogge, Müller und A. Petersen. Dass sich noch am gleichen Abend 27 der versammelten Deutschen in die Mitgliederlisten des noch zu gründenden Vereins eintragen liessen, ${ }^{3}$ mag gleichermassen als Hinweis auf Kleins Rednergabe sowie darauf, dass mit dem Verein ein tatsächlıches Bedürfnis unter den deutschen Arbeitern und Handwerkern in Kopenhagen erfüllt wurde, gelten.

Schon nach einer Woche, am Freitag dem 17. September, konnte die Statutenkommission der zweiten Versammlung deutscher Arbeiter und Handwerker in Kopenhagen ihren Entwurf vorlegen, den die Anwesenden mit einigen Änderungen guthiessen und annahmen. ${ }^{4}$ Anschliessend wählten sie einen provisorischen Vorstand bestehend aus dem 1. Vorsitzenden Klein, dem 2. Vorsitzenden Müller, dem Protokollführer Nolte und dem Kassierer Bagge, womit der Verein gegründet war. ${ }^{5}$ Eine Woche später wurde der provisorische Vorstand um zwei Revisoren und einen Vertreter beim Zentralvorstand der Kopenhagener Gewerkvereine ergänzt. Das letztgenannte wichtige Amt erhielt Simonsen.

Der Zentralvorstand der freien Gewerkvereine in Kopenhagen ( $D e$ frie Fagforeningers Centralbestyrelse $i$ Kjøbenhavn) war 1874 als Resultat der Zentralisierungsbestrebungen verschiedener Gewerkvereine entstanden. ${ }^{6}$ Er sollte den Platz einnehmen, den die dänische IAA-Sektion als Zentralbehörde der dänischen Arbeiterbewegung innegehabt hatte, bis sie im Mai 1872 Opfer der Behördenrepressionen geworden war. In der Nacht vom 4. auf den 5. Mai 1872 waren nämlich

Ebd., Nr 214 (16.9.1875).

2 Ebd.

3 Ebd.

4 Ebd., Nr 221 (23.9.1875).

E Ebd.

- Vergleiche die zwar veraltete, aber noch immer unersetzliche Studie von Henry Bruun, Den faglige Arbejderbevægelse i Danmark indtil Aar 1900, Bd 1, Kopenhagen 1938, S. 416f.; auch die "Politische Ubersicht" im Neuen Sozial-Demokrat, 4. Jahrg., Nr 71 (24.6.1874). 
Louis Pio, der Mann, der die IAA-Sektion im Oktober 1871 gegründet hatte, ${ }^{1}$ und seine beiden Mitarbeiter Povl Geleff und Harald Brix verhaftet worden, weil Pio trotz Polizeiverbots an seiner Aufforderung $\mathrm{zu}$ einer Massendemonstration für streikende Maurer in Kopenhagen festgehalten hatte. ${ }^{2}$ Die drei waren Monate später $2 \mathrm{u}$ langjährigen Zuchthausstrafen verurteilt worden, die dänische IAA-Sektion wurde am 14. August 1873 durch ein Gerichtsurteil aufgelösst. ${ }^{3}$ Damit verloren die Gewerkvereine, die Basisorganisationen der IAA-Sektion, ${ }^{4}$ ihre politische Zentralbehörde und ihren organisatorischen Zusammenhalt. Die dänische Arbeiterbewegung bestand nur noch aus voneinander isolierten gewerkschaftlichen Organisationen. Um diesem Mangel abzuhelfen, der sich vor allem bei Lohnkämpfen und bei der Agitation negativ auswirkte, bildeten am 30. Mai 1874 eine Reihe Kopenhagener Gewerkvereine einen Zentralvorstand. Zum Vorsitzenden dieser neuen Zentralbehörde, in der jeder Gewerkverein wenigstens einen Vertreter unterhielt, ${ }^{\mathbf{5}}$ wählten sie den seit den fünfziger Jahren in Kopenhagen ansässigen deutschen Barbier Ernst Wilhelm Klein, ${ }^{6}$ der sich als Verfasser radikaler Artikel in der Parteipresse einen Namen gemacht ${ }^{7}$ und wahrscheinlich schon bei der Gründung der dänischen IAA-Sektion eine gewisse Rolle gespielt hatte. ${ }^{8}$

In seinem Amte als Vorsitzender des Kopenhagener Zentralvorstandes leistete Klein in nimmermüden Einsatz und unter grossen persönlichen Opfern ein Stück Arbeit, das die dänische Arbeiterbewegung nicht nur wiederaufrichtete, sondern sie auch, durch Vergrösserung und Zentralisierung, nach aussen und innen festigte. ${ }^{9}$ Als

1 Vgl. O. Bertolt, E. Christiansen, P. Hansen, En Bygning vi rejser. Den politiske Arbejderbevægelses historie i Danmark, Bd 1, Kopenhagen 1954, S. $47 \mathrm{ff}$.

2 Ebd., S. $71 \mathrm{ff}$.

${ }^{3}$ Ebd., S. 74f.

4 Die dänische IAA-Sektion beruhte auf sogenannten fachlichen Selitionen (faglige sektioner), in denen die einzelnen Gewerke organisiert waren. Die Wahrnehmung der politischen Aufgaben - z.B. die Aufstellung von Kandidaten zu den Wahlen - oblag dem Zentralkomitee. Vgl. die Organisationsstatuten in Socialisten, 1. Jahrg. (1871), Nr 10 („Love for Foreningen”). Socialisten, zuerst Wochen-, dann Tageblatt, war der Vorgänger von Soc.-Dem.

5 Love for de frie Fagforeningers Centralbestyrelse, Kopenhagen 1875, § 1.

- En Bygning vi rejser, Bd 1, S. 81f.

7 Socialisten (Tageblatt), 1. Jahrg., Nr 30 (16.8.1872).

'Hans-Norbert Lahme, „Zur Vorgeschichte der dänischen IAA-Sektion”, in: IRSH, 19. Jahrg. (1974), S. 63.

- Vergleiche dazu Kleins Korrespondenz im Neuen Sozial-Demokrat, 5. Jahrg,, Nr 135 (14.11.1875). (,Kopenhagen, 8. Nov."), die die genaueste Statistik der dänischen Gewerkvereine in den siebziger Jahren enthält. H. Bruuns Tabellen (a.a.O., S. 576ff. und 595) liessen sich mit Kleins Angaben ergänzen, bzw. korrigieren. 
Louis Pio zusammen mit seinen beiden Leidensgenossen am 8. April 1875 anlässlich des Geburtstages des dänischen Königs amnestiert wurde und am 6. August Kleins Amt übernahm, änderte das an der Position des Deutschen vorerst nichts, da dieser auf dem eigens für ihn geschaffenen Posten eines Sekretärs beim Zentralvorstand die wichtigsten Fäden weiterhin in der Hand behielt. ${ }^{1}$

Die Vertretung des deutschen Vereins beim Kopenhagener Zentralvorstand weist auf seine innige Verflechtung mit der dänischen Arbeiterbewegung hin. Sie war schon auf der Gründungsversammlung vom 17. September 1875 angekündigt worden ${ }^{2}$ und wurde im Paragrafen 20 der Vereinsstatuten festgelegt (Dok. I). Wie die dänischen Gewerkvereine hatte der deutsche Verein das Recht, an den Diskussionen und Beschlüssen des Zentralvorstandes teilzunehmen. Das bedeutete jedoch nicht, dass der Verein gewerkschaftliche Aufgaben wahrzunehmen hatte, im Gegenteil: schon auf der Gründungsversammlung hatte Klein das Verhältnis des deutschen Vereins zu den Gewerkschaften dahingehend definiert, dass er „darauf hinarbeiten [wolle], dass diejenigen Mitglieder, die einem Gewerke angehören, welches einen sozialistisch organisierten Gewerkverein hier in der Stadt besitzt, sich diesem anschliessen" sollen. ${ }^{3}$ Desweiteren wurde im Januar 1876 in einem Aufruf „An die deutschen Gesinnungsgenossen in Kopenhagen" betont, dass der deutsche Verein keine Gewerkschaftspolitik treiben würde (Dok. II).

Klein definierte den Aufgebenkreis des Vereins als politischen: er sollte den dänischen Gewerkvereinen eine „sogenannte politische und soziale Vorschule" 4 sein. Aus diesem Grunde bestimmten die Vereinsstatuten, dass auch nichtdeutsche Mitglieder in den Verein aufgenommen werden konnten (Dok. I). Der Verein hatte mit anderen Worten die Aufgabe, der beinahe ausschliesslich aus Gewerkvereinen bestehenden und sich in gewerkschaftlicher Tagespolitik beschränkenden dänischen Arbeiterbewegung jener Jahre nicht nur die in Kopenhagen lebenden Deutschen als Mitglieder zuzuführen, sondern er sollte die Gewerkvereine auch um ein politisches Element bereichern. Dazu sollten einzig und allein „die zu Gebote stehenden gesetzlichen Mittel” dienen, vor allem "Vorträge und Diskussionen sowohl in politischer, als in sozialer Hinsicht" (Dok. I) und auf "kulturhistorischem Gebiete" (Dok. II). Von der Sprachschule und dem Arbeitsvermittlungsbüro war nicht mehr die Rede.

In einer Gastwirtschaft in der Kopenhagener Innenstadt hatte der

1 En Bygning vi rejser, Bd 1, S. 83f.

2 Soc.-Dem., 2. Jahrg., Nr 221.

${ }^{3}$ Ebd.

4 Neuer Sozial-Demokrat, 5. Jahrg., Nr 135. 
Deutsche social-demokratische Arbeiterverein ein Verkehrslokal. ${ }^{1} \mathrm{Ab}$ 1. Oktober 1875 lag hier für die Mitglieder der Neue Sozial-Demokrat aus, ${ }^{2}$ und hier fanden auch, zumeist am Freitagabend, die wöchentlichen Sitzungen und Veranstaltungen statt, auf die ab September $1875 \mathrm{im}$ Annoncenteil von Social-Demokraten regelmässig in deutscher Sprache hingewiesen wurde und die häufig mit Aufforderungen an die Deutschen in Kopenhagen zum Beitritt verbunden waren. ${ }^{3}$ Klein hatte in der deutschen Parteipresse schon frühzeitig die Gründung des Vereins mitgeteilt und bekanntgegeben, wann und wo in Kopenhagen er regelmässig zu erreichen sei, damit er zureisenden deutschen Genossen behilflich sein könne. ${ }^{4}$ Es scheint, als seien diese Bemühungen um Mitglieder nicht ohne Erfolg geblieben, denn im Oktober 1875 zählte der Verein schon fünfzig Mitglieder. ${ }^{5}$

$\mathrm{Zu}$ diesem Zeitpunkt war die Aufbauphase des Vereins bereits abgeschlossen, sodass Simonsen und Klein sich von den leitenden Ämtern zurückziehen konnten. Der erstgenannte trat auf der Generalversammlung vom Oktober 1875 seinen Posten als Vertreter des Vereins beim Zentralvorstand an Reimeier ab, weil er bei dieser Behörde schon einen allerdings nicht näher bezeichneten Gewerkverein repräsentierte. ${ }^{6}$ Kurz darauf, der genaue Termin liess sich nicht feststellen, legte Klein sein Amt als Vereinsvorsitzender nieder und übergab es dem aus Berlin zugereisten Heinke. ${ }^{7}$ Am 7. Januar 1876 wurden P. Bloch und Malmström mit den Ämtern des 1. und 2. Vorsitzenden betraut, ${ }^{8}$ doch schon am 31. März wurde ein neuer Vorstand gewählt, diesmal mit $\mathrm{O}$. Waschatz als 1. Vorsitzenden. ${ }^{9} \mathrm{Ab}$ November 1876 unterzeichnete schliesslich B. Esch, der letzte Vorsitzende des deutschen Vereins, die Annoncen in der sozialdemokratischen Presse Dänemarks. ${ }^{10}$

\section{II}

Da sie wöchentlich im Annoncenteil von Social-Demokraten angekündigt wurden, sind wir über die Aktivitäten des Deutschen social-demokratischen Arbeitervereins in Kopenhagen recht gut unterrichtet.

1 Soc.-Dem., 2. Jahrg., Nr 209.

2 Ebd., Nr 229 (2.10.1875).

${ }^{3}$ Vgl. z.B. ebd., Nr. 257 (5.11.1875).

4 Neuer Sozial-Demokrat, 5. Jahrg., Nr 116 (1.10.1875).

5 Ebd., Nr 135.

'Soc.-Dem., 2. Jahrg., Nr 249 (27.10.1875).

? Ebd., 3. Jahrg., Nr 8 (11.1.1876); Heinkes Herkunft bezeugt ebd., 2. Jahrg., Nr 269 (19.11.1875).

8 Ebd., 3. Jahrg., Nr 19 (24.1.1876).

${ }^{9}$ Ebd., Nr 77 (31.3.1876), sowie Nr 119 (25.5.1876).

${ }^{10}$ Zuerst ebd., $\mathrm{Nr} 268$ (17.11.1876), zuletzt in Folkeviljen, 1. Jahrg., Nr 11 (12.3.1877). Danach nur noch „Der Vorstand”. 
Abgesehen von Veranstaltungen mehr gesellschaftlicher Art wie Sylvesterabendfeiern und „gemütlichen Zusammenkünften” anlässlich des Jahrestages der Pariser Kommune oder zur Feier von Lassalles Geburtstag, ${ }^{1}$ wurden vorzugsweise Vortragsabende arrangiert, in der Regel mit anschliessender Diskussion. Die bevorzugten Redner waren in den ersten drei Monaten der Existenz des Vereins Klein und Heinke, danach, als der eine seiner zahlreichen übrigen Geschäfte wegen dem deutschen Verein nur noch wenig Zeit opfern konnte und der andere Kopenhagen verlassen hatte, vor allem Bloch.

Unter den verschiedenen Vortragsthemen erfreuten sich besonderer Beliebtheit solche über den Sozialismus in allgemeinen sowie über politökonomische und soziale Themen. Klein hielt beispielsweise einen "Geschichtlichen Vortrag über den Sozialismus" - ein Thema, das zu seinem üblichen Repertoire gehörte ${ }^{2}$ Heinke sprach über „Maschinen und Grossindustrie”, "Der Arbeitsprozess - Die Arbeitskraft”,"3 Simonsen hielt einen Vortrag über „Die Lehre von Darwin”.4 Das Referat eines Vortrages von Heinke vom Januar 1876 mit dem Titel "Der Fetisch-Charakter des Geldes" sei hier in Übersetzung wiedergegeben:

„Der Redner gab eine erschöpfende Erklärung über die erste Entstehung des Geldes; wie die Priester im Mittelalter lehrten, dass man um Geld den Weg ins Himmelreich finden könne; wie später die Kapitalmacht und ihr Missbrauch entstanden, die die Menschheit im Augenblick so hart bedrücken; danach wurde der Tausch- und Gebrauchswert des Geldes erläutert. In der wissenschaftlichen Ökonomie ist man sich bewusst, in wieweit das Geld zu entbehren ist und dass es nichts anderes ist als ein Gebrauchsgegenstand; die gleiche Meinung hat Karl Marx in seinem berühmten Buch 'Das Kapital' ausgesprochen. Der Vortrag [...] dauerte über eine Stunde". ${ }^{5}$

Das zitierte Vortragsreferat ist die einzige Quelle, die eine längere Beschreibung eines der Vereinsabende enthält; zusammen mit den überlieferten Themen der anderen Vorträge und Diskussionsveranstaltungen ergibt sich der Eindruck, dass das Niveau des deutschen Vereins in Kopenhagen dem damals unter deutschen sozialdemokratischen Arbeitern und Handwerkern üblichen entsprach.

Ebenfalls häufig und, nachdem zuerst Esch und danach Bloch den

1 Z.B.: Soc.-Dem., 3. Jahrg., Nr 65 (17.3.1876), und Nr 88 (13.4.1876).

2 Ebd., 2. Jahrg., Nr 227 (1.10.1875).

3 Ebd., Nr 269; Nr 281 (3.12.1875).

4 Ebd., Nr 275 (26.11.1875).

5 Ebd, 3. Jahrg., Nr 19. 
Vorsitz im Verein übernommen hatten, beinahe ausschliesslich, wurden auf den Wochensitzungen die Lehren Ferdinand Lassalles dargestellt und diskutiert. Die Skala des Gebotenen reicht von einem Vortrag über „Den näheren Zusammenhang der Gegenwärtigen Geschichtsperiode mit der Idee des Arbeiterstandes" (Simonsen) ${ }^{1}$ über die Diskussion von „Fichtes politischem Vermächtnis” (Bloch) ${ }^{2}$ zu Vorlesungen mit anschliessender Diskussion aus dem "Zweiten Vortrag" $(\text { Bloch })^{3}$ und der Verlesung des „Arbeiterprogrammes".4 Mitunter wurden auch nur lapidar „Vorlesungen aus Lassalles Broschüren” angekündigt. ${ }^{5}$ Dass hier mehr zum Ausdruck kam, als nur die in jenen Jahren von Lassalle'schen Theoremen durchsetzte Ideologie sozialdemokratischer deutscher Arbeiter und Handwerker, zeigt die Rolle der deutschen Vertreter bei der Organisationsdebatte im Zentralvorstand der Kopenhagener Gewerkvereine. Seit Juli 1874 diskutierten nämlich die Vertreter der im Zentralvorstand repräsentierten Vereine die Errichtung einer sozialdemokratischen Partei, die die augenblicklich nur auf Gewerkvereinen beruhende Organisation ergänzen sollte. ${ }^{6}$ Unter dem Einfluss der deutschen Vertreter geriet die Organisationsdebatte zur Grundsatzdiskussion über den Wert von gewerkschaftlichen Organisationen und zur Auseinandersetzung mit den Theorien Lassalles, dessen populärste Schriften seit 1874 auch in einer billigen dänischen Ausgabe erhältlich waren. ${ }^{7}$ Die Organisationsdebatte war die erste theoretische Debatte in der dänischen Arbeiterbewegung.

Im Dezember 1875 empfahl Simonsen seinen dänischen Kollegen im Zentralvorstand, die politische Arbeit gegenüber der gewerkschaftlichen mehr $\mathrm{zu}$ betonen ${ }^{8}$ - ein Rat, der die gewerkschaftliche Organisation noch nicht in Frage stellte und durchaus mit den Statuten des Zentralvorstands, in denen auch von der Verbesserung der „politischen [...] Stellung” der Arbeiter und von der „Einführung des

1 Ebd., 2. Jahrg., Nr 272 (23.11.1875).

2 Ebd., 3. Jahrg., Nr 35 (11.2.1876).

${ }^{3}$ Ebd., Nr 77.

4 Ebd., 4. Jahrg., Nr 59 (11.3.1877).

S Ebd., 3. Jahrg., Nr 147 (29.6.1876).

- Ebd., 1. Jahrg., Nr 89 (25.8.1874).

71874 erschienen die ersten sechs Hefte der dänischen Ausgabe von Lassalles Werken, im Februar und März 1875 die beiden letzten: Ferdinand Lassalles Skrifter, 1.-6. Heft, Kopenhagen 1874, 7. und 8. Heft, ebd. 1875. Für die Ubersetzung zeichnete Vilhelm Rasmussen verantwortlich, für die Herausgabe der ersten sechs Hefte E. Heinemann, danach der Zentralvorstand. Vgl. Soc.Dem., 2. Jahrg., Nr 37 (13.2.1875). Obwohl die Einzelhefte nur 8 Øre kosteten, verkauften sie sich schlecht. Die Ausgabe musste deshalb mit dem 8. Heft eingestellt werden. Vgl. ebd., Nr 279 (1.12.1875).

8 Ebd., Nr 296 (21.12.1875). 
allgemeinen und direkten Wahlrechts" die Rede war, ${ }^{1}$ in Einklang stand. Heinke, nach Simonsen und Reimeier der dritte Vetreter des Deutschen social-demokratischen Arbeitervereins beim Zentralvorstand, schlug kurze Zeit später eine andere Tonart an: auf der Zentralvorstandssitzung vom 22 . Januar 1876 sprach er sich nicht nur für die Errichtung einer ,starken politischen Organisation” aus, er bezweifelte auch den Wert gewerkschaftlicher Aktivitäten; „Das Gewerkschaftliche habe das Untergeordnete darzustellen und solle am besten verschwinden", ${ }^{2}$ meinte der Deutsche. Bloch, der Vereinsvorsitzende, stiess in das gleiche Horn, indem er kurz darauf den dänischen Arbeitern und Handwerkern mangelndes Interesse an politischer Arbeit und aussergewerkschaftlichen Fragestellungen vorwarf, was Klein zu der Gegenbemerkung veranlasste, die Arbeiter Dänemarks hätten ,den Geist des Sozialismus niemals verleugnet". ${ }^{3}$

Den stärksten Angriff auf die dänischen Gewerkvereine formulierte jedoch der Vertreter des grossen Tabakarbeitervereins Enigheden (Einigkeit) beim Zentralvorstand, der deutsche Arbeiter Stein. Er hatte schon im November 1875 den Gewerkvereinen ihre Befähigung zu politischer Arbeit abgesprochen und solche Ambitionen für ,fromme Wünsche" erklärt. ${ }^{4}$ Auf einer Sitzung des Zentralvorstandes Mitte Januar 1876 hielt Stein ein fünfviertelstündiges, heftiges Plädoyer für eine Neuorganisierung der dänischen Arbeiterbewegung, wie er sie sich vorstellte. Stein machte den in den Ohren seiner dänischen Kollegen sicher unerhört klingenden Vorschlag, die Gewerkvereine sofort aufzulösen und statt ihrer eine politische Partei zu errichten. Als die anderen Vertreter opponierten, nannte er sie eine Versammlung von "Nachtmützen". ${ }^{5}$ Seine drastischen Vorschläge begründete Stein mit Lassalles Kritik an den Streiks als Mitteln auf dem Wege der Emanzipation des Arbeiterstandes und mit dem Hinweis auf die allgemeine Gültigkeit des nur mit politischen Mitteln zu brechenden „Ehernen Lohngesetzes". ${ }^{6}$ Hatten die anderen deutschen Verfechter einer starken politischen Organisation der dänischen Arbeiterbewegung Lassalles Vorstellungen nur durchschimmern lassen, so gab sich Stein hier als Lassalleaner reinsten Wassers zu erkennen.

Die lassalleanische Haltung der deutschen Arbeiter in der Organisationsdebatte fand auch bei einigen dänischen Vertretern im Zentralvorstand Widerhall. So hatte Hansen, der Vertreter der Schmiede,

1 Love for de frie Fagforeningers Centralbestyrelse, a.a.O., Einleitung.

2 Soc.-Dem., 3. Jahrg., Nr 38 (15.2.1876).

3 Ebd.

4 Ebd., 2. Jahrg., Nr 271 (21.11.1875).

' Ebd., 3. Jahrg., Nr 13 (16.1.1876).

${ }^{6}$ Ebd. 
Heinkes Ansichten über den geringen Wert der Gewerkvereine nachhaltig unterstützt, ${ }^{1}$ und auch Foltmar, der Socialdemokratisk Samfund (Sozialdemokratische Gesellschaft) ${ }^{2}$ beim Zentralvorstand repräsentierte, hatte sich den Ansichten der deutschen Kollegen angeschlossen und für eine ausschliesslich politische Organisation der dänischen Arbeiterbewegung plädiert. ${ }^{3}$

Klein und Pio kostete es einige Mühen, dem Treiben der Lassalleaner Einhalt zu gebieten. Foltmar gegenüber meinte Pio, dass die existierende Form der Organisation der dänischen Arbeiterbewegung das Spezifikum des Landes und der ,natürlichen Entwicklung der Arbeiterfrage in Dänemark" entsprungen sei; es sei aus diesen Gründen falsch, der dänischen Arbeiterbewegung fremde Organisationsmodelle aufpfropfen $z u$ wollen. ${ }^{4}$ Klein warnte öffentlich vor ,den falschen Propheten, die [...] unter dem Anschein des fleissigen Einsatzes für die Sache [der Arbeiter] ganz im Gegenteil versuchten, diese zu vernichten". ${ }^{5}$ Entscheidend war jedoch eine längere Rede Pios vor dem Tabakarbeiterverein Enigheden im Februar 1876, im Verlaufe derer er die Bedeutung von politischer und gewerkschaftlicher Organisation im Kampf der Arbeiterklasse erläuterte und zu den Theorien Lassalles Stellung nahm. Pio, der sich auf einen Artikel Johann Philipp Beckers im Vorboten berief, ${ }^{6}$ meinte, es sei falsch und auf keinen Fall im Sinne Lassalles, „seine Meinungen zu Dogmen oder ihn selbst zu einem unfehlbaren Papst" auszurufen. Lassalle selbst, würde er noch leben, hätte sich dagegen verwahrt und seine Lehren den veränderten Bedingungen angepasst. ${ }^{7}$ In der gleichen Rede warnte Pio die Tabakarbeiter eindringlich vor der "seltsamen Sekte der Lassalleaner" in Deutschland, die er der Fehlinterpretation und des Missbrauchs der Lassalle'schen Schriften bezichtigte und die, obschon nur ,eine unbedeutende Partei”, gelegentlich auftauchten, „um die Begriffe zu verwirren und den Fortschritt der Arbeitersache zu verzögern" ${ }^{8}$ Diese

1 Ebd., Nr 38.

${ }^{2}$ Socialdemokratisk Samfund war ein Zwischending zwischen Gewerkverein und politischem Verein, der teilweise einen eigenen Weg zu gehen versuchte. Beispielsweise formulierte dieser Verein schon im September 1875 ein politisches Programm, zehn Monate bevor die dänische Sozialdemokratie ihr "GimleProgramm" verabschiedete. Vgl. En Bygning vi rejser, Bd 1, S. 79f. Das gleichermassen vom Gothaer und vom Eisenacher Programm beeinflusste Programm von Socialdemokratisk Samfund in Samfundet, 1. Jahrg. (1875), Nr 1-2.

${ }^{3}$ Soc.-Dem., 3. Jahrg., Nr 40 (17.2.1876).

4 Ebd.

${ }^{5}$ Ebd., Nr 39 (16.2.1876).

6 Ebd., Nr 33 (9.2.1876); Der Vorbote, 4. Jahrg. (1869), S. 66, 69 f.

' Soc-Dem., 3. Jahrg., Nr 33.

8 Ebd. 
Bemerkungen könnten ein Hinweis darauf sein, dass einige der Deutschen in Kopenhagen, am ehesten Stein, dem Allgemeinen Deutschen Arbeiterverein (Sitz Hamburg) angehörten oder dieser Splittergruppe, die sich 1873 vom ADAV abgespalten hatte, ${ }^{1}$ zumindest nahestanden. ${ }^{2}$ Dafür spricht auch die Überlegung, dass einige der deutschen Arbeiter und Handwerker in Kopenhagen wahrscheinlich aus dem nahegelegenen Hamburg, bzw. aus dem norddeutschen Raum kamen, wo diese Fraktion orthodoxer Lassallegläubiger beinahe ausschliesslich anzutreffen war. ${ }^{3}$

Pios Rede vor den Tabakarbeitern bezeichnete den Höhepunkt der Organisationsdebatte und das definitive Ende der Auseinandersetzungen mit dem Lassalleanismus. Gleichzeitig war es Pio gelungen, Stein vor seinem Gewerkverein zu diskreditieren. Nach weiteren Querelen wurde er im Mai 1876 aus dem Tabakarbeiterverein ausgeschlossen, ${ }^{4}$ und danach verliert sich seine Spur im Dunkeln. Auf dem Kongress von Gimle, dem ersten Kongress der dänischen Arbeiterbewegung vom 6 . bis 8. Juni 1876, wurden schliesslich die Konsequenzen aus der Organisationsdebatte und der Auseinandersetzung mit den Theorien Lassalles gezogen: die auf dem Kongress endgültig gegründete Dänische Sozialdemokratische Arbeiterpartei (Det danske socialdemokratiske Arbejderparti) beruhte, wie die IAA-Sektion, auf den Gewerkvereinen als Basisorganisationen; 5 das Parteiprogramm, welches auf dem Kongress verabschiedet wurde und bis 1888 Gültigkeit hatte, war zwar eine fast sklavische und etwas holprige Übersetzung des Gothaer Programmes, doch war die Passage vom „Ehernen Lohngesetz" ersatzlos gestrichen worden. ${ }^{8}$

\section{III}

Das Bild von Kontinuität in den Aktivitäten des deutschen Vereins, das die wöchentlichen Ankündigungen der Vereinssitzungen im Annoncenteil von Social-Demokraten vermitteln, gerät mit dem Ausgang des Jahres 1876 in Unordnung. Die Annoncen des Vereins, der im Sommer

1 D. Fricke, Die deutsche Arbeiterbewegung 1869-1890. Ihre Organisation und Tätiglkeit, Leipzig 1964, S. 92.

${ }^{2}$ Die Durchsicht der in Frage kommenden Jahrgänge des (Hamburger) SocialDemokrat, Organ zur Vereinigung sämtlicher Arbeiter Deutschlands, ergab jedoch keinerlei Hinweise auf eine solche Verbindung.

${ }^{3}$ D. Fricke, a.a.O., S. 93.

4 Soc.-Dem., 3. Jahrg., Nr 121 (28.5.1876).

5 Der Organisationsstatut ist abgedruckt ebd., Nr 131 (10.6.1876). Eine deutsche Utbersetzung findet sich im Neuen Sozial-Demokrat, 6. Jahrg., Nr 73 (28.6.1876). - Das Gimle-Programm ist abgedruckt in Soc.-Dem., 3. Jahrg., Nr 131. Die deutsche Utbersetzung im Neuen Sozial-Demokrat, 6. Jahrg., Nr 75 (2.7.1876), ist fehlerhaft. 
und Herbst 1876, wohl in Anlehnung an die Neubenennung der vereinigten deutschen Partei in Gotha, gelegentlich als „Deutscher socialistischer Arbeiterverein" figuriert, ${ }^{1}$ erscheinen nun nicht mehr regelmässig einmal in der Woche, sondern mit wochenlangen, oft sogar monatelangen Lücken. ${ }^{2}$ Auf dem Kongress von Gimle war der deutsche Verein nicht vertreten, obwohl er als Mitglied des Zentralvorstandes durchaus $Z$ ugang gehabt hätte. ${ }^{3}$

Die Gründe für den hier offenbar werdenden Niedergang des deutschen Vereines lassen sich nur vermuten. Denkbar ist, dass der Konjunktureinbruch in der zweiten Hälfte der siebziger Jahre, der für die dänische Industrie teilweise katastrophale Folgen hatte und sich vor allem in hohen Arbeitslosenzahlen bemerkbar machte, viele Vereinsmitglieder zum Abwandern gezwungen hat. ${ }^{4}$ Sicherlich spielte auch der Ausschluss Kleins aus der dänischen Arbeiterpartei eine Rolle. Zwischen Pio und Klein hatte es seit der Rückkehr des Gründers der dänischen Arbeiterbewegung aus dem Gefängnis Eifersüchteleien und Auseinandersetzungen gegeben, die am 18. Juni 1876 mit dem Ausschluss des Deutschen endeten. ${ }^{5}$ Ohne Erfolg versuchte Klein, sich gegen die ihm vorgeworfenen Vergehen - u.a. Spionage für die Polizei $^{\mathbf{6}}$ - zu verwahren; sogar seine Landsleute im deutschen Verein ergriffen Pios Partei, der sein Vorgehen gegen den Rivalen mit einer geschickt geführten Hetzkampagne absicherte. ${ }^{7}$ Am 21. Juli 1876 forderte Bloch seinen Landsmann auf, vor dem deutschen Verein Rede und Antwort zu stehen. ${ }^{8}$ Ob Klein dieser Aufforderung nachkam, ist nicht bekannt. Im Laufe des Jahres 1877 verliert sich seine Spur vorläufig im Dunkeln, ${ }^{9}$ jedoch ist seine Anwesenheit in Kopenhagen für das Jahr 1881 wieder bezeugt. ${ }^{10}$

1 Z.B. in Soc.-Dem., 3. Jahrg., Nr 220 (22.9.1876) und in den folgenden Wochen.

2 Z.B. zwischen dem 29. Juni und dem 21. Juli 1876, sowie zwischen dem 21. Juli und dem 14. September 1876.

${ }^{3}$ Vgl. die Teilnehmerliste des Kongresses in Soc.-Dem., 3. Jahrg., $\mathrm{Nr} 129$ (8.6.1876).

4 Schon im März 1875 forderte Klein wegen der grossen Arbeitslosigkeit in Kopenhagen wanderlustige deutsche Genossen auf, die Stadt vorläufig zu meiden; Neuer Sozial-Demokrat, 5. Jahrg., Nr 29 (10.3.1875).

5 Vgl. hierzu z.B. Bruun, a.a.O., S. 234f.

'So in Soc.-Dem., 3. Jahrg., Nr 205 (5.9.1876). Klein dementiert ebd., Nr 206 (6.9.1876).

7 Besonders infam wirkt Pios Artikel „E. W. Klein.”, ebd., Nr 161 (15.7.1876). Kleins Position vertritt die satirische Zeitschrift Figaro, 4, Jahrg. (1876), Nr 22.

${ }^{8}$ Soc.-Dem., 3. Jahrg., Nr 166 (21.7.1876).

- Oluf Bertolt, Pionerer. Mændene fra halvfjerdsernes Arbejderbevægelse, Kopenhagen 1938, S. $202 f$.

10 Der Sozialdemokrat, 3. Jahrg. (1881), Nr 37 (,Aus Dänemark”). Von Klein wird allerdings behauptet, er sei jetzt ,internationaler Spitzel” und stünde „in russisch-dänisch-preussischen Polizeidiensten". 
Der deutsche Verein hielt am 9. April 1877 seine letzte ordentliche Generalversammlung ab. ${ }^{1}$ Für den 22 . April wurde jedoch schon wieder eine Generalversammlung einberufen, diesmal eine ausserordentliche. Die Tagesordnung bestand aus einem einzigen Punkt: „Der Stand und Zweck des Vereins". 2 Diese Annonce ist das letzte Lebenszeichen des Deutschen social-demokratischen Arbeitervereins in Kopenhagen. Es scheint, als habe er sich auf der ausserordentlichen Generalversammlung selbst aufgelöst.

\section{DOKUMENTE}

Statuten des deutschen social-demokratischen Arbeitervereins.

$\S 1$. Zweck des Vereins ist, die social-demokratischen Ideen durch alle $\mathrm{zu}$ Gebote stehenden gesetzlichen Mittel zu verbreiten und das Wohl der Mitglieder in geistiger und materieller Beziehung $\mathrm{zu}$ fördern, besonders durch Vorträge und Diskussionen, sowohl in politischer, als in socialer Hinsicht.

$\S 2$. Ein jeder unbescholtene Mensch, der sein 18. Jahr erreicht hat, kann als Mitglied des Vereins aufgenommen werden, ohne Unterschied der Sprache und Nationalität.

$\S 3$. Mitglieder, die gegen die Zwecke des Vereins handeln, können vom Verein ausgeschlossen werden.

$\S 4$. Jedes Mitglied hat bei Aufnahme in den Verein eine Einschreibegebühr von 25 Öre $z u$ entrichten, wofür ihm Statuten nebst Quittungsbuch eingehändigt werden. Der wöchentliche Beitrag ist 10 Öre. $\S 5$. Wer mit seinem Beitrage 6 Wochen im Rückstande ist, wird vom Verein als ausgetreten betrachtet, und kann nur wieder aufgenommen werden gegen Nachzahlung der Rückstände. Krankheitsfälle oder sonstige unverschuldete Hindernisse ziehen keine Rückstände nach sich.

$\S 6$. Der Vorstand des Vereins besteht aus einem 1. und 2. Vorsitzenden, 1 Schriftführer und 1 Kassierer, welche auf $\frac{1}{2}$ Jahr durch schriftliche Abstimmung mit einfacher Majorität gewählt werden.

Wiederwahl kann Statt finden.

§ 7. Die Wahlen des Vorstandes, sowie zweier Revisoren, finden Statt auf den halbjährigen Generalversammlungen in April und Oktober.

1 Soc.-Dem., 4. Jahrg., Nr 71 (25.3.1877).

2 Ebd., Nr 92 (22.4.1877). 
§ 8. Der erste Vorsitzende hat die Angelegenheiten des Vereins in Übereinstimmung mit dem Vorstande zu leiten.

$\S 9$. Der Vorstand hält eine wöchentliche Sitzung; über alle Verhandlungen des Vorstandes, als auch des Vereins, wird ein Protokoll geführt, welches nur die Verhandlungsgegenstände und deren Abstimmung enthält, aber keinen Auszug der Verhandlungen. Wird ein Beschluss gefasst, gegen welchen ein oder mehrere Mitglieder Verwahrung einlegen, können der oder diese fordern, ihren Protest zu Protokoll zu führen, doch ohne Motivierung.

$\S 10$. Der Vorstand bestimmt die Geschäftsordnung und erlässt die Bekanntmachungen in den öffentlichen Blättern. Wenn ein Mitglied des Vorstandes in 3 auf einander folgenden Sitzungen ausbleibt, wird er als ausgetreten betrachtet.

$\S 11$. Der Kassierer legt jeden Monat seine revidierte Abrechnung dem Vorstande vor und jedes halbe Jahr bei der Generalversammlung. Ohne Einwilligung des Vorstandes kann er Nichts auf Rechnung des Vereins verausgaben; sobald die Umstände es erlauben, kann ihm ein Honorar vom Verein bewilligt werden.

$\S 12$. Der Kassenbestand darf nicht 20 Kronen übersteigen; alles Übrige wird unter Kontroll in ein Bankinstitut niedergelegt.

$\S 13$. Die Generalversammlung hat die höchste Beschlussfähigkeit in allen Vereinsangelegenheiten, Auslegung und Abänderung der Statuten; doch müssen bei lezterer wenigstens $\frac{2}{3}$ der anwesenden Mitglieder dafür stimmen.

$\S 14$. Jährlich werden 2 Generalversammlungen abgehalten, in den Monaten April und Oktober, wo die Wirksamkeit des Vereins, der Kassenbericht und die Zahl der Mitglieder bekannt gemacht wird.

$\S 15$. Jede Generalversammlung muss 14 Tage vorher öffentlich in den Blättern bekannt gemacht werden, ebenso müssen Anträge 8 Tage vor der Generalversammlung mit Namensunterschrift an den Vorstand eingereicht werden.

§ 16. Die Erledigung aller Gegenstände auf der Generalversammlung, mit Ausnahme der Wahlen - $\$ 6$ - geschieht durch einfache Abstimmung; bei Stimmengleichheit wird schriftlich abgestimmt, und giebt dieses denselben Erfolg, geht die Sache aus der Tagesordnung. Die Versammlung ist beschlussfähig, ohne Rücksicht auf die Zahl der Anwesenden.

$\S 17$. Ausserordentliche Generalversammlungen müssen einberufen werden wenn:

a) die Majorität des Vorstandes dies beschliesst;

b) wenn 25 Mitglieder schriftlich darauf antragen.

Für ausserordentliche Generalversammlungen gelten dieselben Bestimmungen, wie bei den ordentlichen. 
$\S 18$. Das Vermögen des Vereins ist während seines Bestehens untheilbar; ausscheidende Mitglieder haben keinen Anspruch darauf. Die Auflösung des Vereins kann nur dann erfolgen, wenn $\frac{1}{4}$ der Mitglieder darauf anträgt, und $\frac{2}{3}$ derselben bei der Urabstimmung sich dafür erklären, in welchem Falle der Vorstand eine Generalversammlung einzuberufen hat, welche diese Angelegenheit zum Austrage bringt.

Dieser Paragraf kann von keiner Generalversammlung geändert werden.

$\S 19$. In zweifelhaften Fällen, wo die Statuten Nichts vorschreiben, ist der Vorstand berechtigt, die nothwendigen Vorkehrungen zu treffen, doch steht er der Generalversammlung zur Verantwortung dafür.

$\S 20$. Der Verein schliesst sich an den Centralverein der freien Gewerkschaften in Kopenhagen und entrichtet die damit verbundenen Ausgaben; ebenso giebt er seinen Beitrag zum allgemeinen Agitationsfond.

Kopenhagen 1875

Abdruck nach dem Original im ABA;

Druckfehler wurden korrigiert.

\section{II}

An die deutschen Socialisten in Kopenhagen.

Parteigenossen! Wie es Euch wohl bekannt sein wird, existiert hier ein deutscher socialistischer Arbeiterverein, der es sich zur Aufgabe gemacht hat, die social-politischen Grundsätze auszubreiten und zur Geltung zu bringen. Wir wissen, dass es hier viele deutsche Socialisten giebt, die an der dänischen Arbeiterbewegung nicht Theil nehmen können, wegen der Unbekanntschaft mit der Sprache und es ist ihnen deshalb in unserm Verein eine Gelegenheit geboten, in ihrer Muttersprache an den Debatten sich zu betheiligen, und da keine Gewerkschaftsinteressen zur Sprache kommen, sondern bloss Gegenstände auf dem kulturhistorischen, socialökonomischen und politischen Gebiete, ersuchen wir Euch, in unsern Verein zu treten, der jeden Freitag Abend seine Sitzung hält im Lokale Købmagergade 10, 1. Saal.

Nehmt Theil am grossen Kampfe der Neuzeit für das Recht der arbeitenden Klassen.

Der Vorstand des deutschen socialdemokratischen Arbeitervereins. 
Indem ich vorbenanntem Aufrufe meine ganze Billigung gebe, erlaube ich mir auch dänische Parteigenossen, die der deutschen Sprache mächtig sind, einzuladen.

Mit Brudergruss

E. W. Klein.

Social-Demokraten, 3. Jahrg., Nr 18 (21.1.1876) 